\title{
Multi-Sensory Media Experiences
}

\author{
Marianna Obrist \\ University of Sussex, UK
}

\begin{abstract}
The way we experience the world is based on our five senses, which allow us unique and often surprising sensations of our environment. Interactive technologies are mainly stimulating our senses of vision and hearing, partly our sense of touch, and the sense of taste and smell are widely under-exploited. There is however a growing international interest of the film, video, and game industries in more immersive viewing and gaming experiences. In the 20th century there was a demand for a controllable way to describe colours that initiated intense research on the descriptions of colours and substantially contributed to advances in computer graphics, image processing, photography and cinematography. Similarly, the 21st century now demands an investigation of touch, taste, and smell as sensory interaction modalities to enhance media experiences.
\end{abstract}

\section{ACM Classification}

H.5.1 [Information Interfaces and presentation]: Multimedia information systems - audio, video

Author Keywords: Multi-sensory interaction; User experience; Interaction design; Sensory integration; Media experiences; TV

\section{Short Bio}

Marianna Obrist is a Lecturer in Interaction Design at the University of Sussex, UK. She is leading the SCHI Lab (Sussex Computer Human Interaction) integrated in the Creative Technology research group established within the School of Engineering and Informatics. Prior to joining Sussex, she was a Marie Curie Fellow at Culture Lab at the School of Computing Science in Newcastle University and

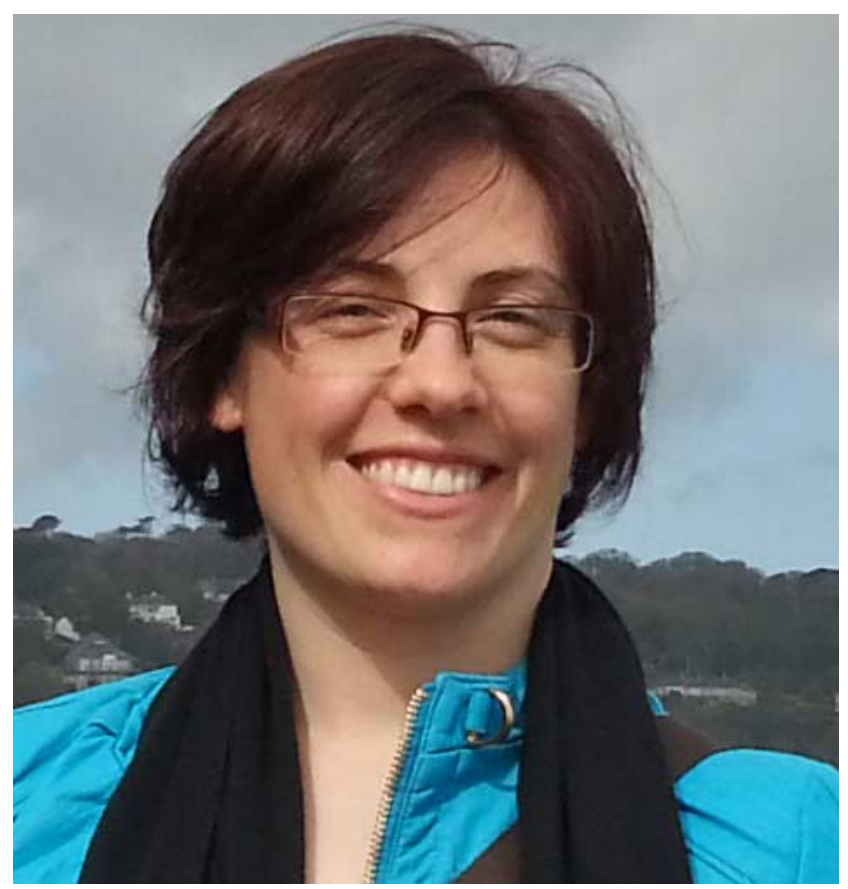

before she worked as an Assistant Professor for HumanComputer Interaction at the Department of Computer Science at the University of Salzburg, Austria. Her current research focuses on the systematic exploration of touch, taste, and smell experiences for human-computer interaction. She has been recently awarded $€ 1.5 \mathrm{Mk}$ by the European Research Council for a five-year project to expand the research into 'Sensory Experiences for Interactive Technologies' (SenseX). Marianna has published widely in the fields of human-computer interaction and user experience, and is currently Vice Chair for the ACM TVX steering committee. 